Check for updates

Cite this: Mater. Adv., 2021, 2, 3871

Received 16th February 2021, Accepted 2nd April 2021

DOI: $10.1039 / \mathrm{d} 1 \mathrm{ma00139f}$

rsc.li/materials-advances

\section{Effect of solvation on the synthesis of MOF-based microrobots and their targeted-therapy applications $\dagger$}

\begin{abstract}
Xueliang Mu, Yukun Zhong, Teng Jiang and U Kei Cheang (D) *
Magnetically driven mobile micro/nanorobots have a significant influence on the application and development of intelligent targeted drug delivery. However, the potential risk of biological toxicity is one of the major problems in drug-loaded micro/nanorobot fabrication. Therefore, there is an urgent need to combine the features (high cargo-loading, low biotoxicity, and good biodegradability) of metalorganic frameworks (MOFs) with micro/nanorobot mobility. In this paper, the concept of green chemical synthesis is used to prepare mass-manufactured biodegradable MOF-based microrobots with low biotoxicity and high drug loading for the targeted treatment of cancer cells. Based on the solvation principle of the binary solvent system, the two-component solvent will be mixed with aprotic polar solvents ( $\mathrm{X}=\mathrm{DMAC}, \mathrm{DMF}, \mathrm{DMSO}, \mathrm{NMP})$ and proton polar solvents $(\mathrm{MeOH})$ to reduce original aprotic polar solvent toxicity. This can adjust the electrophilicity and polarity of the solution environment, change the configuration of organic ligands, and directly affect the nucleation and growth of MOF crystallites. The results show that five different MOF crystal structures can be synthesized on the surface of microrobots. The MOFs synthesized in a $\mathrm{DMAC} / \mathrm{MeOH}$ solvent system have cubic structures with good biocompatibility and drug delivery properties. Furthermore, the magnetically actuated motion of MOF-based microrobots with different geometries was systematically tested to obtain the best swimming performance. Subsequently, the microrobots were guided through vascular-like microfluidic channels and can be precisely controlled. Thus, this establishes a foundation to create massmanufactured microrobotic systems that provide a new direction for small-scale medical robots with low toxicity, high drug loading capacity, biodegradability, and precise motion control.
\end{abstract}

\section{Introduction}

In the past two decades, advances in micro/nanotechnology have facilitated the miniaturization of mobile-medical robots into the micro/nanoscale, enabling the possibility of controlling micro/ nanorobots inside living organisms or microfluidic devices. ${ }^{1} \mathrm{Micro} /$ nanorobots can convert externally applied and/or environmental stimuli (light, ultrasound, magnetic field, electric field, etc.) into motion in order to travel in small spaces and biological environments that are not easily accessible by conventional means. ${ }^{2}$ Because of their small size and controllable mobility, micro/nanorobots hold promise for new ideas for biomedical applications, such as targeted drug and cell delivery, sensing, diagnosis, and microsurgery. To perform these applications safely, current research on micro/nanorobots emphasizes on

Department of Mechanical and Energy Engineering, Southern University of Science and Technology, Shenzhen, 518055, China. E-mail: cheanguk@sustech.edu.cn; Tel: +86-755-88015352

$\dagger$ Electronic supplementary information (ESI) available. See DOI: 10.1039/d1ma00139f using new bio-friendly materials ${ }^{3}$ and non-toxic green manufacturing processes. ${ }^{4}$ As a result, a trend in the manufacturing of biocompatible micro/nanorobots is forming. ${ }^{5}$ However, in the face of the complexity of biological environments, there is a great demand for smaller and safer micro/nanorobots with tunable functional properties for precise biomedical tasks in vivo.

The traditional manufacturing methods, including direct laser writing (DLW) ${ }^{6}$ and glancing angle deposition (GLAD), ${ }^{7}$ are highly effective at creating the structures for micro/nanorobots; the next consideration for biomedical micro/nanorobots must focus on obtaining the surface properties that are optimal for drug delivery. Among pore surface engineering methods, the synthesis of metal-organic frameworks (MOFs) with highly tunable features on the surface of micro/nanorobots is a promising way to increase their surface functionality. As a unique host/guest matrix, a MOF allows controllable integration of the properties of metal ions and organic ligands to obtain different characteristics, such as high porosity and chemical functionalities. ${ }^{8,9}$ In the context of micro/ nanorobot-enabled drug delivery, the self-assembly of organic ligands with metal ions or clusters on micro/nanorobots yields a 
large number of binding sites, which greatly improves their drug-carrying capacity. A zeolite imidazole framework (ZIF) ${ }^{10}$ is a MOF subfamily, whose crystal structure is similar to that of a zeolite. In the ZIF series, zinc-based ZIF or ZIF-8 is an ideal drug carrier $^{11}$ for high loading capacity and controlled release due to its high porosity, biodegradability, and low cytotoxicity.

The typical MOF synthesis process allows for crystal polymorphism $^{12}$ to create special MOF microcrystals that can be used for heterogeneous catalysis, drug delivery, and other applications. Inspired by this, many methods ${ }^{13}$ have been proposed to control the particle size and morphology of MOF; one of the most convenient means is by changing the solvent system. ${ }^{14} \mathrm{MOF}$ synthesis usually requires aprotic and polar solvents to dissolve enough organic linkers; ${ }^{15}$ unfortunately, many of these solvents, including the traditional members $N, N$-dimethylformamide (DMF), $N, N$-dimethylacetamide (DMAC), $N$-methylpyrrolidone (NMP) and dimethyl sulfoxide (DMSO), ${ }^{16}$ have been noted for their apparent reproductive toxicity and the need for green alternatives has been repeatedly emphasized. Therefore, it is essential to reduce potentially toxic substances in MOFs and develop more sustainable synthesis routes using low-toxic solvents instead of biologically and environmentally harmful solvents. ${ }^{17}$

Herein, we will modify the ZIF-8 heterostructures on the surface of microrobots using solvation-based methods with low toxic solvent systems. In other words, the composition changes in the binary solution system (aprotic polar solvents/proton polar solvents) would coordinate the interaction between the ZIF-8 crystal grains and the microrobots, thereby forming various tunable MOF micro/nanostructures on the surface of the microrobots. The twocomponent solution mixes polar protic solvents and polar aprotic solvents in different ratios as an experimental variable. The experimental results show that the crystal morphology can be determined according to the proportion of the two-component solvents during crystal growth. When the mixing ratios (MeOH to $\mathrm{X}, \mathrm{X}=\mathrm{DMAC}$, DMF, DMSO, or NMP) change from 0:10 to 10:0 (vol:vol), the final shape of ZIF-8 crystals can change from micro-cubic to nano-cubic, cheese-like, rod-like, and flake-like. Among these forms, the microrobots in certain binary solution systems, such as DAMC:MeOH (4:6), DMF:MeOH (1:9), DMSO:MeOH (1:9), and NMP:MeOH (1:9), will yield MOF crystals with prominent cubic characteristics and appropriate size for micron-level robots. The solvent systems here have low toxicity and are suitable for green synthesis. The magnetically actuated MOF-based microrobots prepared with DAMC: $\mathrm{MeOH}(4: 6)$ were tested to have acceptable biological toxicity and excellent drug loading performance. In this work, a specific-crystalline MOF-based microrobot can integrate the functions of high drug loading and precise motion control, which is ideal for precise transportation of therapeutic molecules to the diseased areas through wireless magnetic control.

\section{Experimental}

\section{Chemicals}

All solvents and reagents were purchased from Aladdin and can be used directly without further purification, including methanol
(MeOH, 99.5\%), $\mathrm{N}, \mathrm{N}$-dimethylacetamide (DMAC, 99.5\%), $\mathrm{N}, \mathrm{N}$ dimethylformamide (DMF, 99.5\%), dimethyl sulfoxide (DMSO, 99.5\%), $N$-methyl-2-pyrrolidone (NMP, 99.5\%), $N$-octanol (OCT, 99.5\%), 2-methylimidazole (98\%), propanone (99.5\%) and isopropanol (IPA, 99.7\%). Deionized water used in experiments was purified from Milli-Q ${ }^{\circledR}$ Direct-Q 5 UV.

\section{Microrobot fabrication}

Photoresist sacrificial platforms were created on which microrobots would be fabricated using photolithography. A silicon ( $\mathrm{Si}$ ) wafer was used as the substrate. The substrate was immersed in propanone and sonicated for $10 \mathrm{~min}$ and then washed with IPA and DI water. To pattern the microrobots, a layer of photoresist (AZ 5214) with a thickness of about $2 \mu \mathrm{m}$ was coated on the $\mathrm{Si}$ substrate using a spin coater. The sample was prebaked at $65{ }^{\circ} \mathrm{C}$ for $60 \mathrm{~s}$ and at $95{ }^{\circ} \mathrm{C}$ for $180 \mathrm{~s}$. After $20 \mathrm{~min}$ of cooling at room temperature, the sample with a photomask was placed in a UV exposure unit (Mask Aligner, SUSS MA6) for pattern transfer through photolithography. The UV-exposed sample was baked at $95{ }^{\circ} \mathrm{C}$ for $60 \mathrm{~s}$, developed for $40 \mathrm{~s}$ in a SU-8 developer, and hard baked at $150{ }^{\circ} \mathrm{C}$ for $20 \mathrm{~min}$. Afterward, photoresist sacrificial platforms were obtained. Masks with different designs were used to create microrobots with different shapes.

To create microrobots on the AZ 5214 platforms, first, a $200 \mathrm{~nm} \mathrm{ZnO}$ thin film was deposited on the AZ 5214 structures using confocal radio frequency (RF) magnetron sputtering. The sputtering system (KYKY-500CK-500ZF) uses 4 inch ZnO, Ni, and $\mathrm{Al}$ composite targets (Beijing Goodwill Metal, 99.9\%). The silicon substrate containing the sample was mounted on a 6 inch alloy substrate. Sequentially, $\mathrm{Al}, \mathrm{Ni}$, and $\mathrm{Al}$ thin films with the respective thicknesses of $10 \mathrm{~nm}, 300 \mathrm{~nm}$, and $10 \mathrm{~nm}$ were deposited onto the $\mathrm{ZnO}$ thin film. The deposition was performed under the conditions of $40{ }^{\circ} \mathrm{C}, 1.33 \mathrm{~Pa}$ sputtering gas pressure, and $50 \mathrm{sccm}$ Ar flow rate, and the RF power used for the $\mathrm{ZnO}$ deposition process and the $\mathrm{Ni}$ and $\mathrm{Al}$ deposition process was $150 \mathrm{~W}$ and $100 \mathrm{~W}$ respectively. After that, another $200 \mathrm{~nm} \mathrm{ZnO} \mathrm{film}$ was deposited onto the Al layer using the same aforementioned ZnO sputtering process and conditions. The base pressure of all samples was higher than $20 \mu \mathrm{Pa}$. The rotation speed of the alloy substrate was set at $18 \mathrm{rpm}$ for uniform thickness. Finally, the designed magnetic microrobots consisting of $\mathrm{ZnO} / \mathrm{Al} / \mathrm{Ni} / \mathrm{Al} / \mathrm{ZnO}$ will be ready for MOF preparation.

\section{MOF-based microrobot preparation}

A high-temperature annealing process was used to carbonize AZ 5214 under the microrobot, which is helpful for the separation of the microrobot from the photoresist. The prepared sample was placed in a muffle furnace with an annealing temperature of $410{ }^{\circ} \mathrm{C}$ for the SU-8 separation treatment of the microrobot. The isothermal time was 5 hours and the heating rate was $10{ }^{\circ} \mathrm{C} \mathrm{min}{ }^{-1}$. After annealing, the microrobots were released via ultrasound treatment.

ZIF-8 based microrobots were fabricated by growing ZIF-8 $\mathrm{MOF}$ on the released $\mathrm{ZnO} / \mathrm{Al} / \mathrm{Ni} / \mathrm{Al} / \mathrm{ZnO}$ microrobots. Numerous experiments were performed to test the effect of solvents on the morphology of the MOF formed. In each experiment, $2 \mathrm{~g}$ of 
2-methylimidazole was dissolved in a closed container $(100 \mathrm{~mL})$ with $50 \mathrm{~mL}$ of two-component solvent at ambient temperature. The two-component solvent consists of $\mathrm{MeOH}$ and $\mathrm{X}$ (X = DMAC, DMF, DMSO, NMP, or OCT) in a volume ratio of $0: 10$ to 10:0 (vol:vol). Using hydrothermal synthesis, the $\mathrm{ZnO} / \mathrm{Al} / \mathrm{Ni} / \mathrm{Al} / \mathrm{ZnO}$ microrobots on the substrate was mixed in the reaction mixture at $65{ }^{\circ} \mathrm{C}$ for 2 days; after this process MOF-based microrobots were formed. Next, the MOF-based microrobots underwent an activation process using a repeated two-step procedure: (1) the MOF based microrobots were immersed in fresh methanol solvent $(\mathrm{MeOH})$ at ambient temperature for $1 \mathrm{~h}$ to exchange solvent and unreacted molecules; and (2) microrobots were collected using a magnet, dried in an oven at $150{ }^{\circ} \mathrm{C}$, and immersed again in $\mathrm{MeOH}$ for $1 \mathrm{~h}$. This process was repeated 3 to 5 times. After the solvent exchange, the microrobots were placed in a $150{ }^{\circ} \mathrm{C}$ vacuum oven overnight, collected, and further tested. The sample nomenclature is based on the volume ratio of solvent component, for example, the sample prepared in the binary solvents $(\operatorname{vol}(\mathrm{MeOH}): \operatorname{vol}(\mathrm{DAMC})=4: 6)$ would be denoted as DAMC6, and other samples are named accordingly.

\section{Sample characterization}

The crystal structures of the MOFs were verified by X-ray diffraction (XRD, RIGAKU Smartlab, Japan) at $45 \mathrm{kV}$ and $200 \mathrm{~mA}$ by $\mathrm{Cu}-\mathrm{K} \alpha$ radiation with wavelength $\lambda=1.54 \AA$. The scanning range of $2 \theta$ was $5-50^{\circ}$ with a step of $0.02^{\circ}(2 \theta)$, and the scanning speed was $3^{\circ}(2 \theta) \mathrm{min}^{-1}$.

Scanning electron microscopy (SEM, Zeiss Merlin, Germany) and energy dispersive spectrometry (EDAX, octane number Pro) were used to characterize the morphology, particle size, and elemental analysis. The accelerating voltage of the electron beam is $3 \mathrm{keV}$ and the current is $100 \mathrm{nA}$ for morphology. The accelerating voltage of the electron beam is $15 \mathrm{keV}$ and the current is $1 \mathrm{pA}$ for elemental analysis.

The attenuated total reflection infrared (ATR-IR) spectra using a PerkinElmer UATR (single reflection diamond) were recorded to analyze the functional groups of the MOF-based microrobots.

The initial $\mathrm{pH}$ value of mixed reactants was measured with a pH meter (PHS-25, Lei-Ci).

\section{Cell culture}

L929 and Hela 229 cells were provided by the Stem Cell Bank at the Chinese Academy of Sciences. The cells were grown in MEM Complete Medium (containing 90\% of no glucose MEM (GIBCO), $10 \%$ of $(\mathrm{v} / \mathrm{v})$ fetal bovine serum (FBS, GIBCO), and $1 \%$ of (v/v) penicillin/streptomycin (10000 $\mathrm{U} \mathrm{mL}^{-1}$, GIBCO)). The cells were maintained at $37^{\circ} \mathrm{C}$ in a humidified atmosphere of $5 \% \mathrm{CO}_{2}(\mathrm{v} / \mathrm{v})$.

\section{Cell viability}

The live L929 and Hela 229 cells were dispersed by soluble cytosolic enzymes (trypsin-EDTA (0.25\%), phenol red, GIBCO), then seeded in 48 well microplates at a concentration of $1 \times$ $10^{5}$ cells with $500 \mu \mathrm{L}$ MEM no glucose complete medium/well and stabilized in an incubator for $24 \mathrm{~h}$. Subsequently, MOF based microrobots with concentrations of $14 \mu \mathrm{g} \mathrm{mL}{ }^{-1}$ and $28 \mu \mathrm{g} \mathrm{mL}{ }^{-1}$ were added to the L929 and Hela 229 cell culture, respectively. After $48 \mathrm{~h}$ culture time, the cells were incubated with WST-8 (a water-soluble tetrazolium salt) solution at $37{ }^{\circ} \mathrm{C}$ for $2 \mathrm{~h}$. The spectrophotometric absorbance of WST-8-formazan produced by the dehydrogenase activity in living cells was measured at a wavelength of $450 \mathrm{~nm}$ using an Infinity $\mathrm{M}$ nano (Tecan). The spectrophotometric absorbance measured by this assay is highly correlated with the number of living cells. After the data collection, the cell microplates were washed once with sterile PBS, and then incubated in no glucose MEM complete medium.

\section{Loading of doxorubicin hydrochloride (DOX)}

Prior to drug loading, $5 \mathrm{mg}$ of MOF-based microrobots were activated by degassing at $180{ }^{\circ} \mathrm{C}$ overnight. After activation, the impurities were removed from the pores, allowing the drug to be adsorbed freely. The microrobots were then suspended in $10 \mathrm{~mL}$ of doxorubicin hydrochloride (DOX) solution $\left(1 \mathrm{mg} \mathrm{mL}{ }^{-1}\right)$ and shaken at ambient temperature for 5 hours to ensure that equilibrium is reached. During the 5 hour period, $100 \mu \mathrm{L}$ of supernatant was collected every $15 \mathrm{~min}$. The amount of adsorbed DOX was calculated indirectly by monitoring the reduction of the UV-vis spectrum of the drug in aqueous solution to study the drug's adsorption kinetics. The drug concentration was calculated from the absorption peak at $\lambda=482 \mathrm{~nm}$ to evaluate the dose. The calibration curve is displayed in Fig. S1 (ESI $\dagger$ ). By subtracting the value of drug loading efficiency (LE) from the DOX amount initially loaded, the MOF-based microrobot drug loading amount can be derived. LE was obtained using the following formula: drug loading efficiency $(\%)=$ the amount of drug loaded/the amount of initial drug $\times 100 \%$.

\section{Swimming control of MOF-based microrobots}

By applying a magnetic torque, $\tau$, the MOF-based microrobots will be rotated along its easy axis. A uniform rotational magnetic field generated using three pairs of Helmholtz coils was used to actuate the microrobot's swimming motion, as shown in Video S1 (ESI $\dagger$ ). The torque on a magnetic microrobot in a magnetic field is given by:

$$
\vec{\tau}=\mu_{0} V \vec{M} \times \vec{H}
$$

where $V$ and $M$ are the volume and magnetization of the microrobot, respectively. $\mu_{0}$ is the permeability of free space and $H$ is the applied field. The maximum magnetic field strength $\left(B=\mu_{0} H\right)$ would not exceed $4 \mathrm{mT}$ with the optimal frequency. To actuate the microrobot, the field vector $B$ is rotated in a plane perpendicular to the easy axis. The MOF-based microrobots were placed inside an open chamber, where the bottom of the chamber is a clean silicon wafer substrate. The chamber was placed in the Helmholtz coil center to ensure a uniform magnetic field. Controlling the rotational frequency and the planar orientation of the rotating magnetic field will alter the swimming velocity and heading direction of the microrobots, respectively, which in turn will enable individual or group control of the MOF-based microrobots to accomplish tasks within the microfluidic channel. 


\section{Microfluidic channel fabrication}

The SU-8 microfluidic channel was fabricated through photolithography. A layer of SU-8 2150 (a thickness of $200 \mu \mathrm{m}$ ) was spin-coated $(2000 \mathrm{rpm})$ on a $3^{\prime \prime}$ silicon wafer, followed by softbaking at $65{ }^{\circ} \mathrm{C}$ and $95{ }^{\circ} \mathrm{C}$ for 10 and 45 min respectively, exposure to UV light at a dose of $220 \mathrm{~mJ} \mathrm{~cm}^{-2}$, and post-exposure baking at $65{ }^{\circ} \mathrm{C}$ and $95{ }^{\circ} \mathrm{C}$ for 10 and $45 \mathrm{~min}$ respectively. Finally, open microfluidic channels were obtained after development using a SU-8 developer and drying at $180{ }^{\circ} \mathrm{C}$ for $1 \mathrm{~h}$. Each SU-8 microfluidic device was air plasma treated (Harrick Plasma Inc) for $30 \mathrm{~min}$ to clean the surface.

\section{Results and discussion}

\section{Morphology of a MOF-based microrobot surface}

Four common low-toxic solvents were used to study the crystallization behavior of ZIF, and the experimental conditions for the growth of each specific crystal form were repeated. In the solvothermal synthesis of ZIF-8, the volume ratio of the two components in the solvent will affect their particle size and morphology. In general, the particle size depends on the nucleus and growth rate. A higher nucleation rate corresponds to smaller particles; this means the particles grow very fast and eventually stop growing when the ingredients are exhausted. Before changing the solvent composition, the solution system will provide the initial excess ligand concentration to ensure a stable initial environment and efficient forwarding reactions. As a variable, the polar aprotic solvent $\mathrm{X}$ is added to the solvent system to compensate for the one-component solvent system (such as a single polar and electrophilic system), and then try to adjust the nucleation and growth rate. As shown in Fig. 1, in pure methanol solution $(\mathrm{MeOH})$, ZIF-8 nanoparticles grow parallel on the $\mathrm{ZnO}$ film without agglomeration. As the proportion of other components (X) increases, the grain size of ZIF-8 first increases and then decreases. In the DMAC/MeOH binary solvent, cubic ZIF crystal particles are present in a wide range of volume ratios (10-90\%). The presence of ZIF-8 appears as nanosheets or rods only when the DMAC concentration is very low or high. In the DMF/MeOH binary solvent system, only $10 \%$ (volume fraction) of DMF presents a typical ZIF-8 crystal cubic structure. As the ratio increases, ZIF crystals show cheese-like structures (DMF4), nanoparticles (DMF5 and DMF7), and irregular cubic structures (DMF10).

Similarly, the DMSO/MeOH binary system had different grain morphologies at different volume ratios. For example, very low or high DMSO concentrations can form cubic-shaped ZIF crystals with a wide range of particle sizes. DMSO4 and DMSO5 produce interpenetrating twins of different sizes. DMSO7 would produce flake-like shapes similar to other high-concentration binary solvents. Interestingly, $\mathrm{NMP} / \mathrm{MeOH}$ can produce samples with a single appearance; except for the nanocubic shapes in the NMP1 solvent system; this binary system will only produce flakes

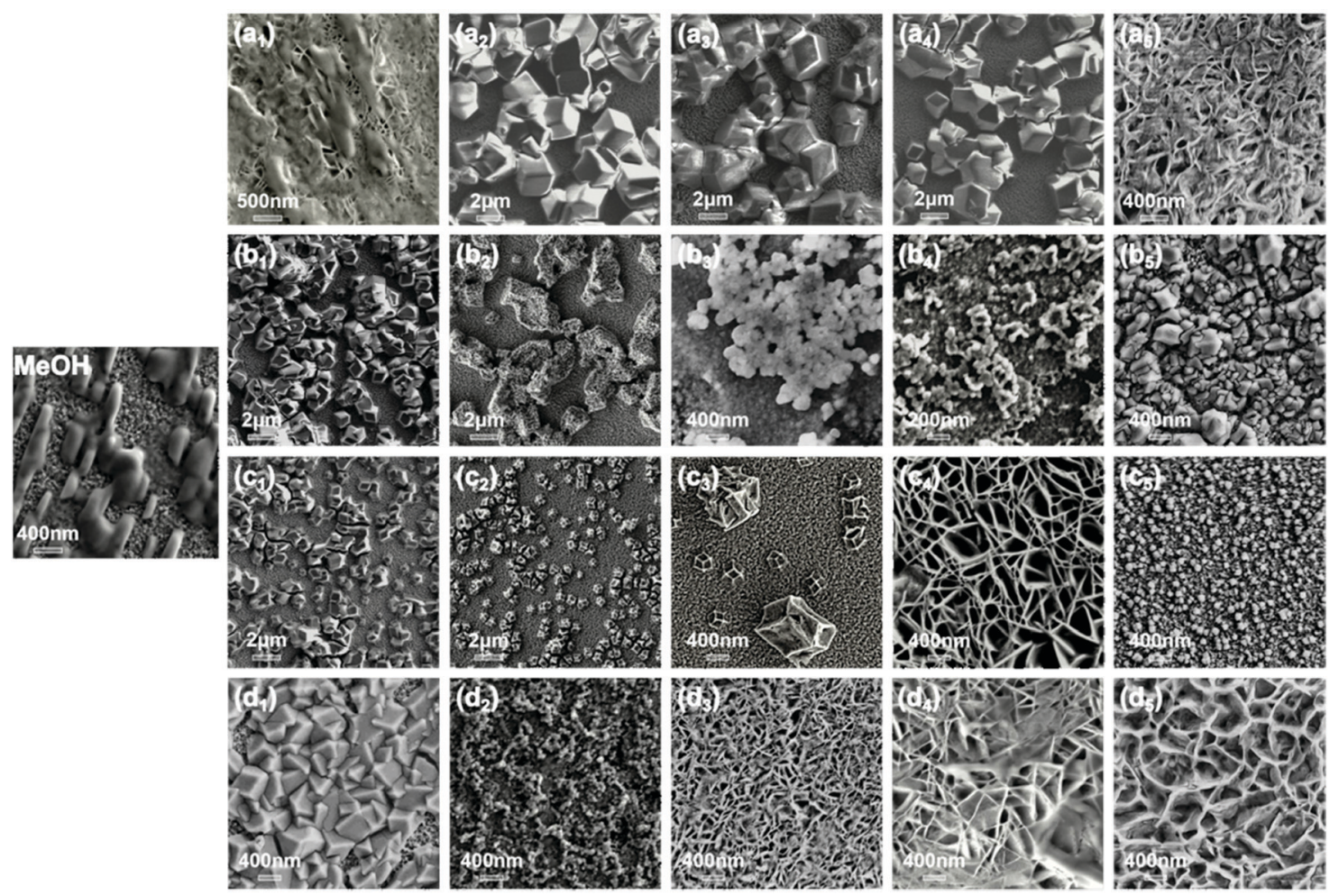

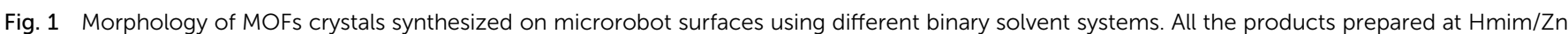

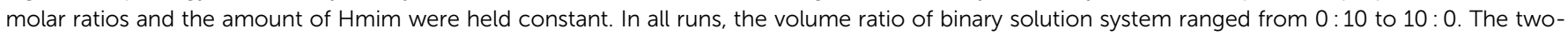

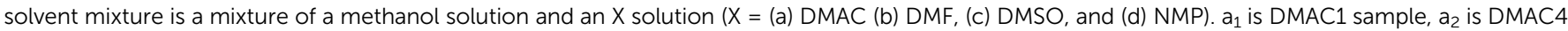

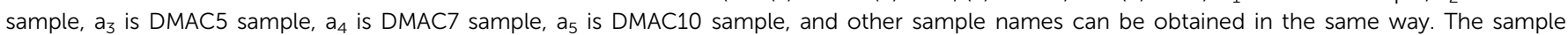
nomenclature is $\mathrm{X}$ solution and volume content, i.e., DMAC1 is a two-solvent mixture with $10 \%$ of DAMC. 
in other volume ratio ranges. It is hypothesized that different twocomponent solvent systems can promote or effectively inhibit the ZIF-8 crystal patterns during the coordination and may seriously affect the formation and polymorphisms of the compound.

\section{Physicochemical properties in different solvent systems}

The addition of the $\mathrm{X}$ solvent component to the two-component solvent system will cause the $\mathrm{pH}$ of the Hmim solution to rise linearly, as shown in Fig. 2(a); the $\mathrm{pH}$ gradient change order is DMSO $>$ DMAC $\geq$ DMF $>$ NMP. The results correspond to the order of dielectric constant (i.e. polarity) of each solvent, ${ }^{18}$ namely DMSO $>$ DMAC $>$ DMF $>\mathrm{MeOH}>$ NMP. However, the MOF complexity order is DMAC $>$ DMF $>$ DMSO $>$ NMP, which could be related to the electrophilic properties (i.e., acceptor number, AN) of the solution. ${ }^{19}$ The order of the number of receptors induced by the solution system was NMP $<$ DMAC $<$ DMF $<$ DMSO (AN or electrophilic). The lower the AN, the more favorable the formation of contact ion pairs. ${ }^{19}$ In other words, the suitable polarity of mixed solvents can enhance the ionization and coordination of Hmim, and the corresponding electrophilic properties can accelerate the forward reaction efficiency of the complex. Therefore, the surface topography diversity of MOF-based microrobots is due to the synergistic effect of solution polarity and electrophilic properties.

It was confirmed that the purpose of the two-component solvent system is to increase the degree of deprotonation of organic zinc ligands, thus increasing the solvation of solution ions. This process can be described as $\mathrm{Hmim}+\mathrm{X} \rightleftharpoons$ $\mathrm{mim}^{-}{ }_{\text {(deprotonation) }}+\mathrm{XH}^{+}$(protonation) $\mathrm{X}$ is a two-component solvent system. The aprotic polar solvent improves the stability of hydrogen cations and mim anions. The dissociation of more $\mathrm{NH}$ groups will increase the alkalinity of the solution, which will also accelerate the corrosion of sacrificial $\mathrm{ZnO}$ to promote the release of zinc ions. Under appropriate alkaline conditions higher than the dissociation constant, $\mathrm{mim}^{-}$of the nitrogen atom is coordinated with $\mathrm{Zn}$ ions of adjacent units to form an assembly structure. Therefore, the balance between the dissolution rate of zinc ions and the coordination rate is essential for forming a clear interface between the microrobot and MOF.

The MOF particles with different shapes yielded from different solvent systems in Fig. 1 were mainly due to the different conformations of $\mathrm{mim}^{-}$in solution and solvent. The ligand mim $^{-}$is highly symmetric and has two equivalent mutants and one $\pi$ electron in the imidazole ring. In the methanol solution,
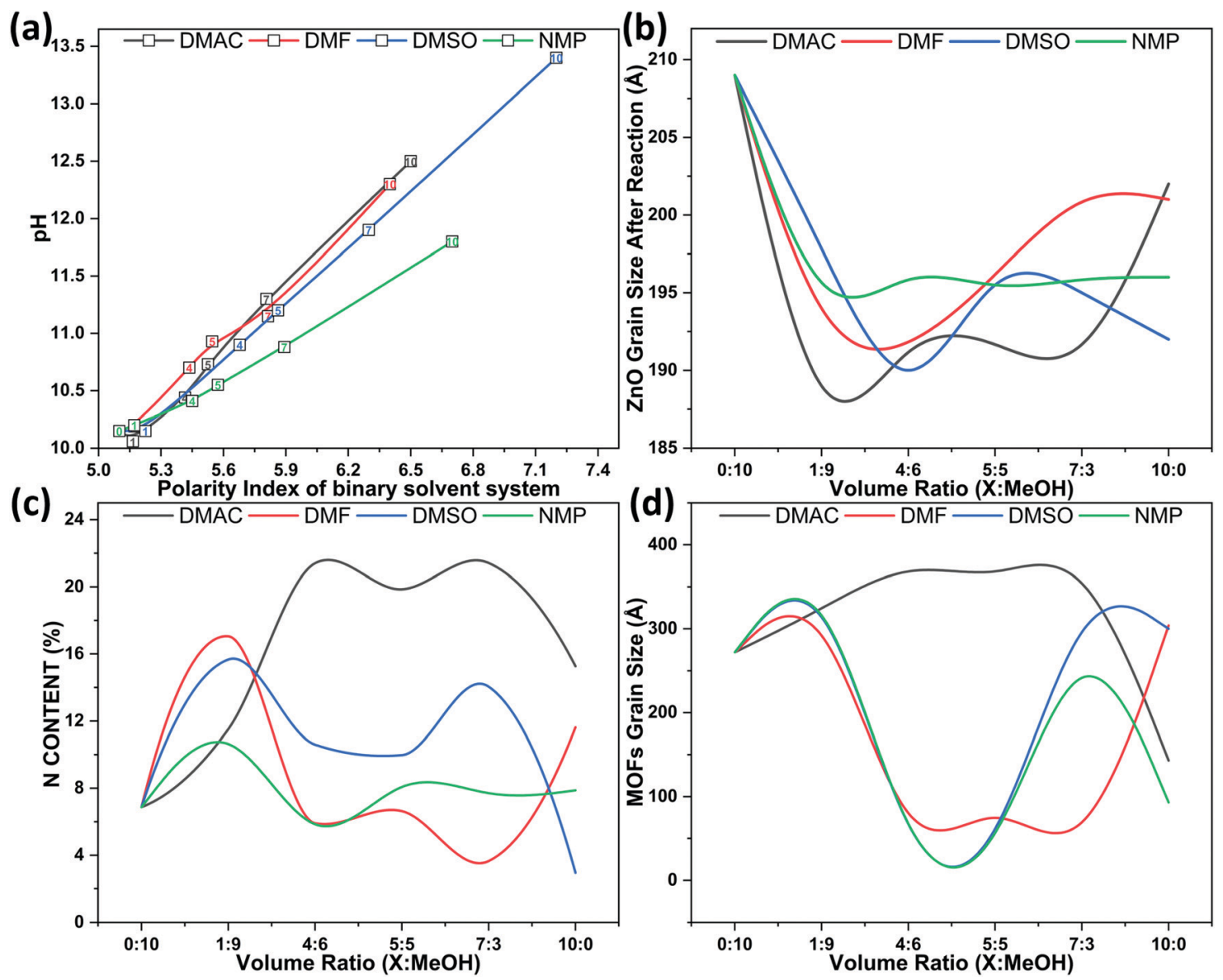

Fig. 2 Volume ratio of the binary solvent system versus (a) $\mathrm{pH}$ value of mixed solution, (b) ZnO grain size after reaction, (c) $\mathrm{N}$ content of MOFs crystal, (d) MOFs grain size. All the products prepared at $\mathrm{Hmim} / \mathrm{Zn}$ molar ratios and the amount of Hmim were held constant in all runs, and the volume ratio of binary solution system ranged from $0: 10$ to $10: 0$. The black, red, blue, and green lines represent methanol solutions containing DMAC, DMF, DMSO, and NMP, respectively. 
$\mathrm{mim}^{-}$will be stacked in the presence of $\pi-\pi .^{20}$ The results show that MOF grains have regular parallel distribution, without observable agglomeration into large particles. Because the nitrogen atoms and single electron pairs of DMAC, DMF, and NMP can form a $\mathrm{p}-\pi$ conjugation system with the carbonyl group, solvent molecules can have strong conjugation with MIM. With the addition of aprotic polar solvents, the hydrogen bond between Hmim and the tight stacking state between imidazole rings will be destroyed immediately. However, due to the steric hindrance effect, ${ }^{19}$ i.e., $\mathrm{NMP}>$ DMAC > DMF, the MOF structures begin to change from cubes to sheets as the concentration of NMP increases. However, the steric hindrance of DMAC becomes weaker, which makes the morphology of MOFs maintain the cubic shape in a wide range of concentrations. DMSO, although not conjugated, has better steric hindrance than $\mathrm{DMF}$, thus the corresponding binary solvent concentrations of DMF and DMSO will produce similarly shaped products. Therefore, the binary solvent system not only regulates the etching rate of the metal layer and the binding of the metal ions in the solvent to the organic ligand at the reaction level, but also adjusts the spatial arrangement of the individual molecules dissolved in the solvent at the structural level, thus guiding the construction of the MOF monomers in different systems into different final MOF grain shapes.

The $\mathrm{N}$ content of various MOF-based microrobots was further studied through SEM/EDX analysis (Fig. 2(c)). The EDX results show that the larger the MOF particle size, the higher the nitrogen content; the peak of $\mathrm{N}$ content change corresponds to micro-cubic MOF. The nitrogen content of rodshaped MOF is usually higher than that of flake-like MOF, but micro-cubic MOF have the highest nitrogen content. In other words, micro-cubic MOF have a richer basic unit structure. When the $x$ component is from $40 \%$ to $70 \%$, MOFs with similar nitrogen content but different shapes can be grown on the $\mathrm{ZnO}$ sacrificial layer. It is worth noting that $5: 5$ volume ratio is not the optimal ratio in the comparative experiment.

According to the FWHM (full width at half maxima) of diffraction peak, the amount of $\mathrm{ZnO}$ and MOF particle sizes on the $\mathrm{ZnO}$ sacrificial layer after corrosion were calculated using Scherrer equation. As shown in Fig. 2(b), when the $\mathrm{X}$ solvent concentration is $10 \%$, the $\mathrm{ZnO}$ sacrificial layer has the highest degree of corrosion. As the volume ratio of $\mathrm{X}$ increases, the corrosion degree of $\mathrm{ZnO}$ begins to decrease, but it is still higher than that of pure methanol solution. Therefore, aprotic polar solution can affect the conformational environment and deprotonation state of organic ligand molecules and adjust the corrosion degree of $\mathrm{ZnO}$ to a certain extent. Fig. 2(d) shows the size changes of MOF particles made from two solvents. Generally, almost all two-component solvent systems have two peaks. The first peak appears in $40 \%$ of DMAC, but the first peak of DMF, DMSO, and NMP is $30 \%$ earlier.

Interestingly, the second peak corresponds to $70 \%$ of each solvent system. In addition, ZnO with a particle size greater than $195 \mathrm{~nm}$ is related to nanoparticle MOFs. If the ZnO particle size is about $195 \mathrm{~nm}$, the MOF particles will be flakes. For ZnO particles from $192 \mathrm{~nm}$ to $195 \mathrm{~nm}$, the MOF particles are micro-cubic. MOFs grown on ZnO particles below $192 \mathrm{~nm}$ are rod-shaped or cheese-like. Compared with the micro-cubic shape, other shapes of MOFs have lower nitrogen content and smaller particle size. Based on the abovementioned synergistic effect of polarity and electrophilicity, it is speculated that the large size of $\mathrm{ZnO}$ was due to insufficient corrosion of $\mathrm{ZnO}$ and insufficient release of zinc ions. As a result, the crystal nucleation rate in the sacrificial layer was faster than the crystal growth rate. In contrast, if the $\mathrm{ZnO}$ particle size is too small, a large number of zinc ions will be released into the $\mathrm{ZnO}$ sacrificial layer. Some free zinc ions entered the solvent because they are released too quickly, while others nucleate in the sacrificial layer to form rod-like or cheese-like structures. Due to insufficient metal ions in the sacrificial layer, the microcubic structures may be incomplete in both cases. Therefore, a suitable two-component solvent system can provide appropriate crystal grain implantation sites and the correct number of metal ions to form a complete MOF structure after etching.

The XRD changes of ZIF-8 synthesized on the microrobot surface using various binary solvent systems are compared in Fig. 3(a).
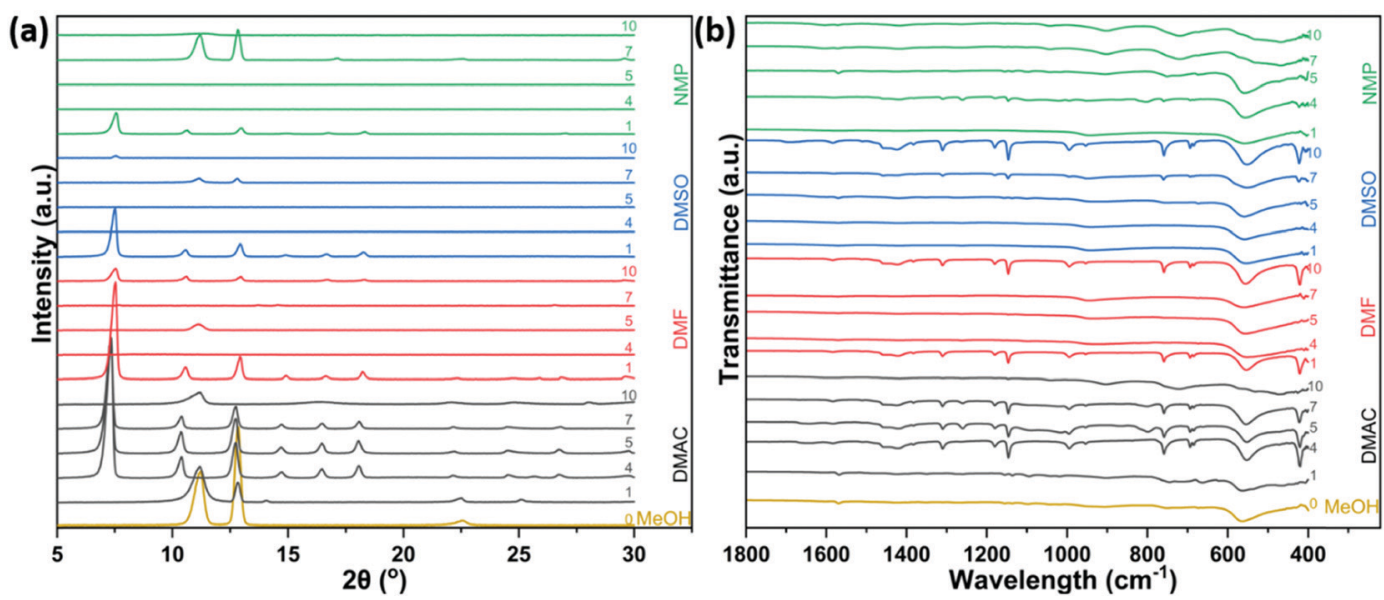

Fig. 3 (a) PXRD patterns and (b) FT-IR spectra of synthesized samples in different ratios of binary solution system. The black, red, blue, and green lines, respectively, represent methanol solutions containing DMAC, DMF, DMSO, and NMP. The yellow line represents pure methanol solution or methanol solution without X-component solvent. 
When the volume ratio of $\mathrm{x}$ component solvent is low, significant $2 \theta$ reflections $\left[7.5^{\circ}(110), 10.4^{\circ}(200)\right.$, and $\left.12.8^{\circ}(211)\right]$ were observed that correspond to the characteristic peak of microcubic ZIF-8. ${ }^{21}$ When the solvent volume ratio of component $\mathrm{X}$ is more than $40 \%$, the diffraction of samples (DMF, DMSO, and NMP) gradually weakens at a reflection peak of $7.4^{\circ}$, where the peaks of (200) and (211) begin to increase. Combined with the SEM results, the significantly enhanced (200) peak produces flake-like MOFs, while the jointly enhanced (200) and (211) peaks evolve into rod-like MOFs. It is worth noting that the diffractions of some samples are too weak to be observed directly due to the presence of overly small or very dispersed MOF particles.

The chemical functional groups of the products prepared in different two-component solvent systems were studied using infrared spectroscopy. As shown in Fig. 3(b), the band fingerprint regions at $687 \mathrm{~cm}^{-1}$ and $754 \mathrm{~cm}^{-1}$ were related to the out-ofplane bending ${ }^{22}$ in the imidazole ring, whose in-plane bending ${ }^{22}$ corresponded to the region at $900-1350 \mathrm{~cm}^{-1}$. The $1678 \mathrm{~cm}^{-1}$ and $1581 \mathrm{~cm}^{-1}$ peaks were caused by the bending and stretching $\mathrm{N}-\mathrm{H}$ vibration, ${ }^{23}$ respectively. The $1425 \mathrm{~cm}^{-1}$ curve is related to the plane stretching ${ }^{22}$ of the imidazole ring. $\mathrm{Zn}-\mathrm{O}$ vibration bands ${ }^{24}$ are detected at 489, 798, and $1023 \mathrm{~cm}^{-1}$ for all samples. As shown in the ESI, $\dagger$ the $\mathrm{Zn}-\mathrm{N}$ stretching (near $420 \mathrm{~cm}^{-1}$ ) peak ${ }^{23}$ intensity change of each sample is consistent with the difference of $\mathrm{N}$ content of each sample (Fig. 2(c)), where the more obvious $\mathrm{Zn}-\mathrm{N}$ peak in the product represents higher $\mathrm{N}$ content. In addition, the FT-IR spectrum of the flake or rod-like MOFs with the lowest $\mathrm{Zn}-\mathrm{N}$ peak intensity is similar to the cubic one. In other words, the basic structural units of all products are identical, but the flake and rod-like MOFs do not have all the spectral characteristics of the micro-cubic MOF.

Hypothetically, these two structures may remain in a transition state during the formation of ZIF-8 crystals. Based on the abovementioned synergistic effect of polarity and electrophilicity, these two shapes mainly appear in low polarity and high electrophilic environments. A low polarity environment reduced the concentration of deprotonated Hmim. In an electrophilic environment, the repulsion between molecules dispersed a large number of deprotonated mim ions, which were completely enclosed in solvent molecule clusters. These conditions directly or indirectly reduced the free ligand groups, thus slowing down the phase rate and terminating the formation of larger ZIF particles during the growth process.
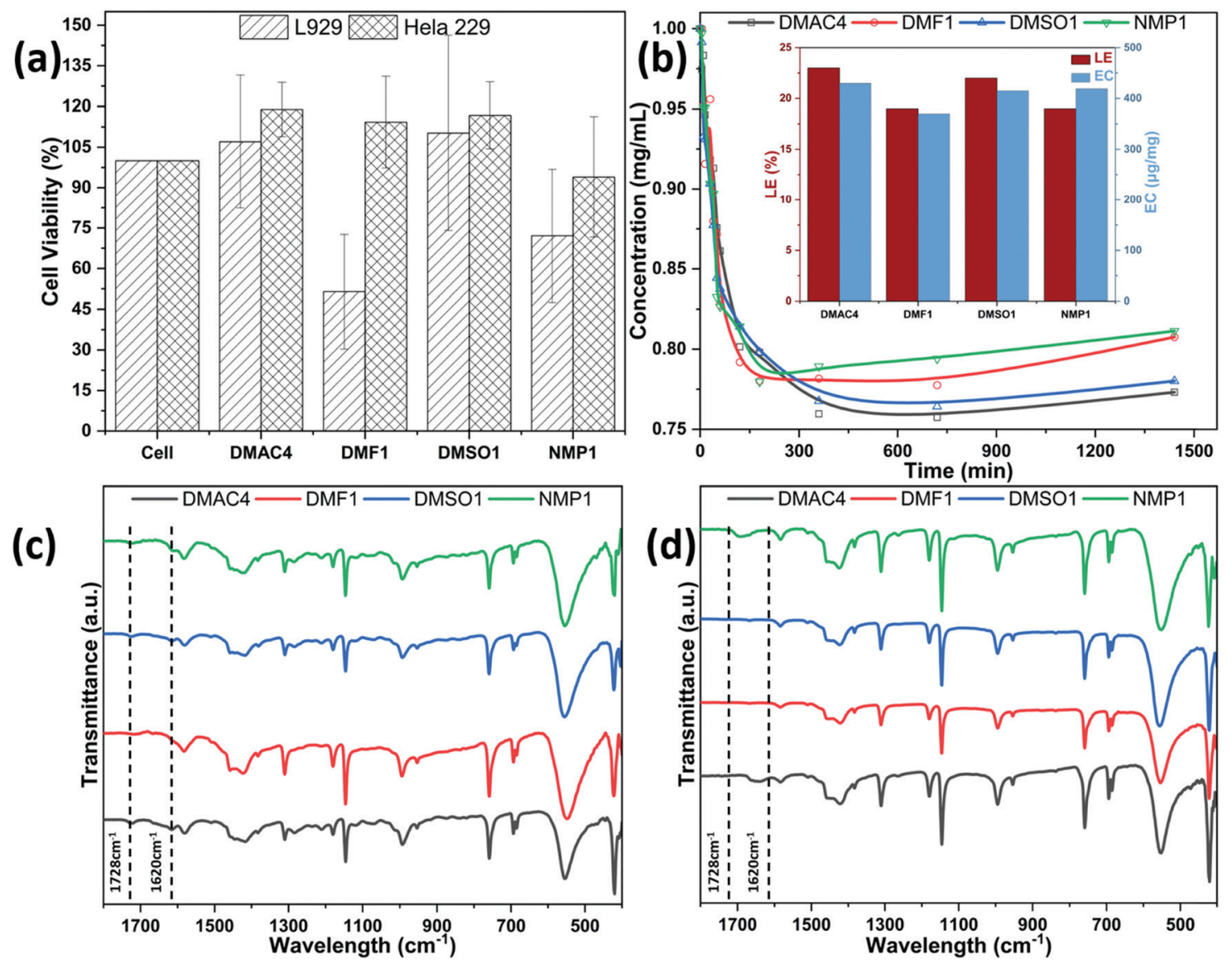

Fig. 4 Biomedical performance testing of MOFs-based microrobot. (a) Cytotoxicity test of L929 and Hela 229, (b) static drug adsorption performance of DOX, and the LE and EA of different samples was concluded from these results in insert graph, (c) FTIR of MOFs-based microrobot after drug adsorption, (d) FTIR of MOFs-based microrobot before drug adsorption. 


\section{Toxicity and drug loading of MOF-based microrobots}

To determine the biocompatibility of MOF-based microrobot materials prepared in different binary solvent systems, cell viability was measured on the surface of MOF-based microrobots mixed with Cell Counting Kit-8 (CCK-8). Fig. 4(a) shows the
CCK-8 results using L929 and Hela 229 cells. The cells were treated with the MOF-based microrobots as the experimental group whereas untreated cells were used as controls. After $48 \mathrm{~h}$, the CCK-8 assay was performed to evaluate the optical intensity of WST-8-formamide between the experimental group and the control group. The results
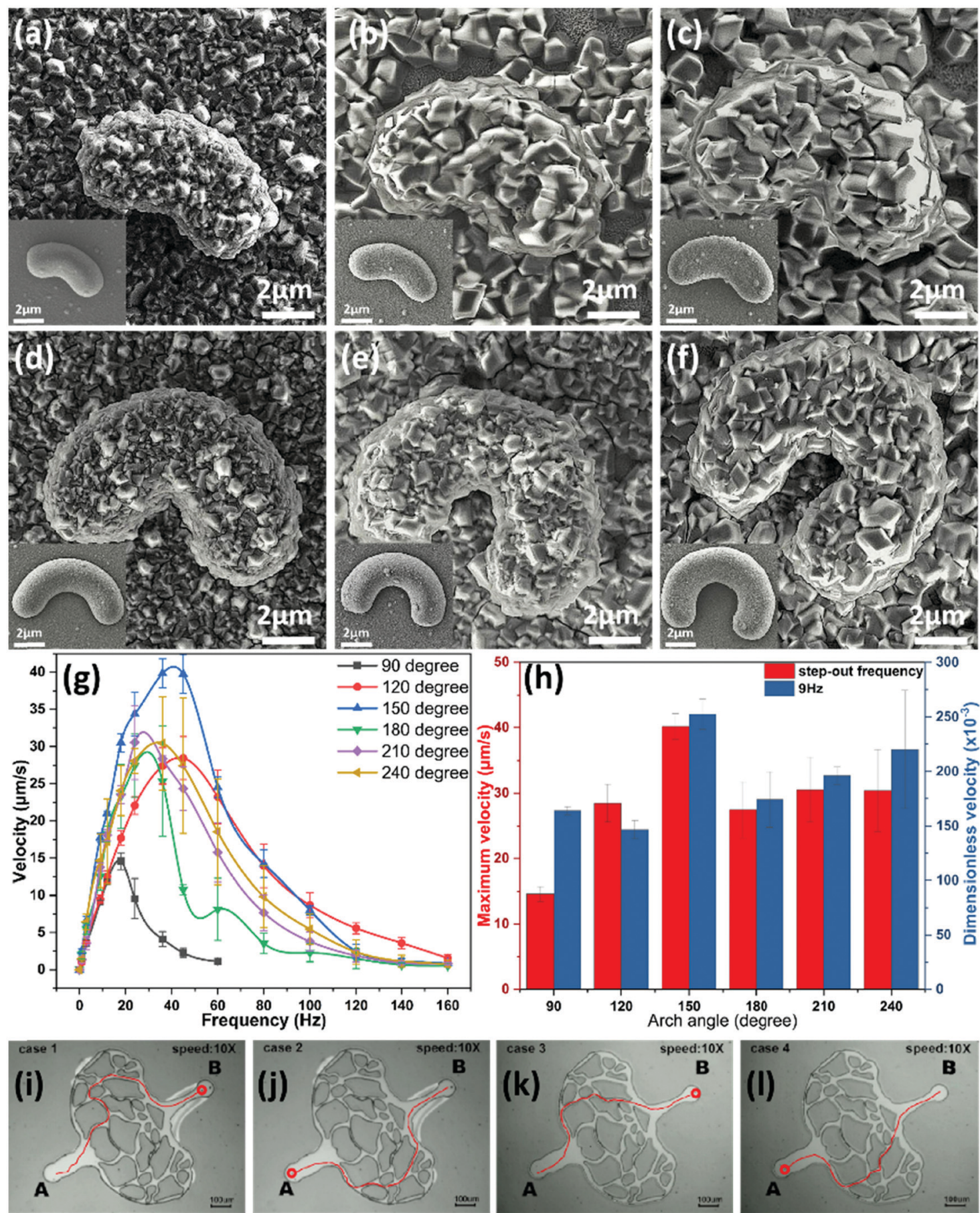

Fig. 5 Arch-like MOFs-based microrobot synthesized in DMAC4 of binary solvent system. SEM images of microrobots with arch degree at (a) $90^{\circ}$, (b) $120^{\circ}$, (c) $150^{\circ}$, (d) $180^{\circ}$, (e) $210^{\circ}$, and (f) $240^{\circ}$. The insets are microrobots without MOFs. (g) Velocity profile vs. frequency of each arch-like microrobot. (h) Maximum velocity and dimensionless velocity plotted against arch degree. (i)-(l) Microrobots magnetically steered to travel in four different paths in a vessel-like microchannel. Note that the trajectories (red lines) were drawn by the after effect (AE). The maximum speeds of the six types of microrobots were recorded in Video S1 (ESI $\dagger$ ), and the navigation videos of different paths were recorded in Video S2 (ESI $\dagger$ ). 
show that Hela 229 had better tolerance to MOF-based microrobots than L929 cells. Besides, the cell viability of the MOF-based microrobot prepared in DMAC and DMSO after 48 hours of cultivation is higher than that of the control group, while those of samples prepared in DMF and NMP are lower than that of the control group. That is, the cytotoxicity trend of MOF-based microrobots is DMAC4 $\leq$ DMSO1 $<$ NMP1 $\leq$ DMF1 to both cells. This may be due to more toxic guest molecules and self-destructive decomposition products in NMP1 and DMF1.

To confirm the drug loading efficiency (LE) and encapsulation capacity (EC) of the MOF-based microrobots, experiments were conducted to measure the concentration of the supernatant yielded from adding around $5 \mathrm{mg}$ of MOF-based microrobot to $1 \mathrm{mg} \mathrm{mL} \mathrm{m}^{-1}$ of DOX, where DOX was detected at an absorption wavelength of $482 \mathrm{~nm}$. As the immersion period increases, the supernatant concentration of the two groups of samples was gradually diluted, and the absorption of the DOX solution reaches a dynamic equilibrium after $300 \mathrm{~min}$. As depicted in the inset graph of Fig. 4(b), DMAC4 has a higher drug loading capacity $\left(\mathrm{LE}=23 \%, \mathrm{EC}=430 \mu \mathrm{g} \mathrm{mg}^{-1}\right)$ than the others (DMF1: $\mathrm{LE}=19 \%, \mathrm{EC}=370 \mu \mathrm{g} \mathrm{mg}{ }^{-1} ;$ DMSO1: $\mathrm{LE}=22 \%, \mathrm{EC}=$ $415 \mu \mathrm{g} \mathrm{mg}^{-1}$; NMP1: $\left.\mathrm{LE}=19 \%, \mathrm{EC}=419 \mu \mathrm{g} \mathrm{mg}^{-1}\right)$. Compared with the FTIR spectrum of DOX-unloaded MOF-based microrobots, the DOX-loaded MOF-based microrobots have an additional absorption band corresponding to the $\mathrm{C}-\mathrm{O}-\mathrm{CH}_{3}$ stretching bands of DOX at $1728 \mathrm{~cm}^{-1}$ and the $-\mathrm{C}=\mathrm{O}-$ stretching band (amide I) at $1620 \mathrm{~cm}^{-1},{ }^{25}$ which confirms the successful loading of DOX on the MOF-based microrobots. Therefore, the microrobots prepared with MOFs in DMAC and DMSO have excellent potential in biomedical applications owing to their enhanced biocompatibility and high drug loading capacity.

\section{Swimming test of MOF-based microrobots}

Six types of arch-like MOF-based microrobots with different arch degrees were synthesized under DAMC4 conditions, as shown in the SEM image in Fig. 5(a)-(f). All of the microrobots have the same width of $2 \mu \mathrm{m}$, which can be seen in the insets of Fig. 5(a)-(f). The surfaces of every arch-shaped microrobot were densely covered with MOF particles of about $1 \mu \mathrm{m}$ in size; as a result, the MOF-based microrobots have thicker widths compared with their "no-MOFs" counterparts in the insets. The swimming performances of the six microrobots were tested via speed tests and steering tests. For the speed tests, the microrobots were subjected to a $3.5 \mathrm{mT}$ magnetic field at different frequencies. Representative microrobots swimming at their respective maximum speeds are shown in Video $\mathrm{S} 1$ (ESI $\dagger$ ). As shown in Fig. 5(g), as the magnetic field frequency increases, all microrobots' speeds tend to increase linear and then decrease when they reach their respective step-out frequencies. After step-out, their speeds start to decrease gradually. The average step-out frequency of the $90^{\circ}$ arch microrobots is the lowest (about $20 \mathrm{~Hz}$ ), and their average maximum speed is also the lowest. The $120-240^{\circ}$ microrobots have similar average performance, but with slightly different maximum speeds and step-out frequencies. It can be seen in Fig. 5(h) that the degree of arc has a significant effect on the robot's kinematic ability. When the robot arc degree was increased from 90 to 150 degrees, the maximum swimming speed increased from $14.59 \mu \mathrm{m} \mathrm{s}^{-1}$ to $40.16 \mu \mathrm{m} \mathrm{s}^{-1}$. However, when the arc degree continued to increase to 240 degrees, the maximum swimming speeds decreased to about $30 \mu \mathrm{m} \mathrm{s}^{-1}$. When different robots were at the same frequency, the 150-degree robot had the best dimensionless swimming speed $\left(252.43 \times 10^{-3}\right)$, which is comparable to the dimensionless speed of the previously reported planar microrobots. ${ }^{26}$ For the steering test, the 150-degree MOF-based microrobots steered to move between points A and B in a complex vessel-like microfluidic channel, as shown in Fig. 5(i)-(l). The microrobot was able to complete the journey 4 times, each time using a different path. The results showed that the MOF-based microrobots could effectively perform the corresponding targeted control in complex and narrow microfluidic channels.

\section{Conclusions}

In summary, this study shows that high quality ZIF-8 particles with different shapes can be synthesized on the surfaces of mass-manufactured magnetic microrobots using different twocomponent solvent systems. The results show that the size and shape of the generated ZIF-8 particles are mainly controlled by the degree of protonation of Hmim in the two-component solvent system. In methanol solutions containing DMAC4, DMF1, DMSO1, or NMP1, micro-cubic ZIF-8 particles with high crystallinity could be obtained on the surfaces of microrobots. Biocompatibility and drug loading tests were performed on the samples prepared using the four mixed solutions. The results consistently showed that the MOF-based microrobots prepared using DMAC4 had good biotoxicity and high DOX drug loading capability. We also performed a detailed evaluation of the mobility of the MOF-based microrobots prepared using DMAC4 and demonstrated their capability for magnetically guided targeted therapy. Finally, we believe that this green and simple synthesis strategy can be widely applied to the large-scale production of nontoxic drugloaded MOF-based microrobots for biomedical applications.

\section{Author contributions}

Dr. Xueliang Mu was mainly responsible for experimental design and operation, and wrote the whole article. Mr. Teng Jiang was responsible for the design of magnetic control and microrobots. Ms. Yukun Zhong was responsible for some characteristic tests and drug loading tests. Prof. U Kei Cheang was responsible for providing suggestions on the experimental process and article revision.

\section{Conflicts of interest}

There are no conflicts to declare.

\section{Acknowledgements}

This work was funded by the National Natural Science Foundation of China (NSFC, 51850410516), the Ministry of Science and Technology of the People's Republic of China (MOST, BG20190232001), the Shenzhen Science and Technology 
Innovation Commission (JCYJ20180302174151692), and the Shenzhen Peacock Plan (20181119590C) awarded to U Kei Cheang. The authors would like to acknowledge the SUSTech Core Research Facilities (SCRF) for technical support.

\section{Notes and references}

1 M. Luo, Y. Feng, T. Wang and J. Guan, Adv. Funct. Mater., 2018, 28, 1706100.

2 J. Li, B. E.-F. de Ávila, W. Gao, L. Zhang and J. Wang, Sci. Robot., 2017, 2, eaam6431.

3 W. Chen, Y. Wen, X. Fan, M. Sun, C. Tian, M. Yang and H. Xie, J. Mater. Chem. B, 2021, 9(4), 1030-1039.

4 R. Arvidsson and S. F. Hansen, Environ. Sci.: Nano, 2020, 7, 2875-2886.

5 A. Terzopoulou, X. Wang, X. Z. Chen, M. Palacios-Corella, C. Pujante, J. Herrero-Martín, X. H. Qin, J. Sort, A. J. deMello and B. J. Nelson, Adv. Healthcare Mater., 2020, 9, 2001031.

6 A. Nishiguchi, A. Mourran, H. Zhang and M. Möller, Adv. Sci., 2018, 5, 1700038.

7 A. Ghosh and P. Fischer, Nano Lett., 2009, 9, 2243-2245.

8 X. Mu, Y. Chen, E. Lester and T. Wu, Microporous Mesoporous Mater., 2018, 270, 249-257.

9 X. Mu, S. Liu, Y. Chen, U. K. Cheang, M. W. George and T. Wu, Ind. Eng. Chem. Res., 2020, 59, 5808-5817.

10 B. Chen, Z. Yang, Y. Zhu and Y. Xia, J. Mater. Chem. A, 2014, 2, 16811-16831.

11 I. B. Vasconcelos, T. G. da Silva, G. C. Militão, T. A. Soares, N. M. Rodrigues, M. O. Rodrigues, N. B. da Costa, R. O. Freire and S. A. Junior, RSC Adv., 2012, 2, 9437-9442.
12 N. Stock and S. Biswas, Chem. Rev., 2012, 112, 933-969.

13 R. Seetharaj, P. Vandana, P. Arya and S. Mathew, Arabian J. Chem., 2019, 12, 295-315.

14 K. A. S. Usman, J. W. Maina, S. Seyedin, M. T. Conato, L. M. Payawan, L. F. Dumée and J. M. Razal, NPG Asia Mater., 2020, 12, 1-18.

15 S. Leubner, R. Stäglich, J. Franke, J. Jacobsen, J. Gosch, R. Siegel, H. Reinsch, G. Maurin, J. Senker, P. G. Yot and N. Stock, Chem. - Eur. J., 2020, 26, 3877-3883.

16 L. Cseri and G. Szekely, Green Chem., 2019, 21, 4178-4188. 17 P. A. Julien, C. Mottillo and T. Friščić, Green Chem., 2017, 19, 2729-2747.

18 A. Jouyban and S. Soltanpour, J. Chem. Eng. Data, 2010, 55, 2951-2963.

19 M. Zohuriaan-Mehr, K. Kabiri and M. Kheirabadi, J. Appl. Polym. Sci., 2010, 117, 1127-1136.

20 F. Cesare Marincola, C. Piras, O. Russina, L. Gontrani, G. Saba and A. Lai, ChemPhysChem, 2012, 13, 1339-1346.

21 K. S. Park, Z. Ni, A. P. Côté, J. Y. Choi, R. Huang, F. J. UribeRomo, H. K. Chae, M. O'Keeffe and O. M. Yaghi, Proc. Natl. Acad. Sci. U. S. A., 2006, 103, 10186-10191.

22 Y. Hu, H. Kazemian, S. Rohani, Y. N. Huang and Y. Song, Chem. Commun., 2011, 47, 12694-12696.

23 M. Zhang, Q. G. Shang, Y. Q. Wan, Q. R. Cheng, G. Y. Liao and Z. Q. Pan, Appl. Catal., B, 2019, 241, 149-158.

24 X. B. Yang, Z. D. Wen, Z. L. Wu and X. T. Luo, Inorg. Chem. Front., 2018, 5, 687-693.

25 Q. Yuan, J. Shah, S. Hein and R. D. Misra, Acta Biomater., 2010, 6, 1140-1148.

26 S. Tottori and B. J. Nelson, Small, 2018, 14, 1800722. 